NASA Technical Memorandum 105352

ICOMP-91-26

IN 34

39203

P. 14

\title{
Stable Localized Patterns in Thin Liquid Films
}

Robert J. Deissler

Institute for Computational Mechanics in Propulsion

Lewis Research Center

Cleveland, Ohio

and

Alexander Oron

Technion-Israel Institute of Technology

Haifa, Israel

November 1991

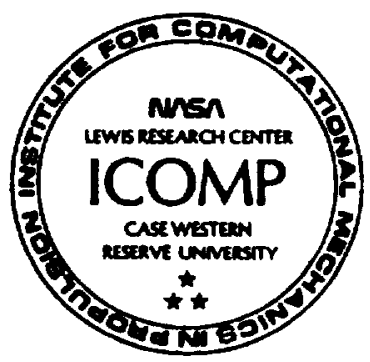

( $\because A 5 A-T A-105352)$ STAPL $=$ LUCALTPL PATTERNS

$192-14322$

IV THIA LIUIO FILMS (NASA) 14 P C GL COO 
Ever since the discovery of solitons, ${ }^{1}$ there has been a great deal of interest in the existence of spatially localized solutions in nonlinear systems. In addition to the solitons of integrable systems, spatially localized solutions have been found in systems with dissipation such as the complex Ginzburg-Landau equation for systems near an inverted bifurcation ${ }^{2-4}$ and a nonlinear phase equation. ${ }^{5,6}$ On a somewhat different footing, we point out that nonlinear evolution equations which result from perturbation expansions have been found to be very useful in studying the dynamics of many physical systems. An example of such an equation is the well-known Kuramoto-Sivashinsky equation which describes systems such as liquid film flow ${ }^{7-9}$ and flame front propagation. ${ }^{10}$ Another such equation which results from similar derivations $s^{1-13}$ is an equation which describes the fluid-air interface of a thin liquid film on the surface of a horizontal plate subjected to a vertical temperature gradient, which gives rise to the Marangoni effect. The Marangoni effect is found to be very important in low gravity conditions and therefore for processes of space technology. ${ }^{12}$ The one-dimensional form of the equation has been found to exhibit stable localized solutions. ${ }^{13}$ However, the existence of stable localized solutions for the two-dimensional equation is still an open question.

In this Letter we: 1) show that the two-dimensional evolution equation, describing the three-dimensional spatiotemporal behavior of the air-liquid interface, has a Liapunov functional that is strictly nonincreasing in time and; 2) exploit this fact to demonstrate the existence of stable axisymmetric localized solutions for a wide range of parameter values. We find that for given parameter values in the equation, the stable localized solutions exist for a wide range of amplitudes and that there is a minimum amplitude below which the solution is no longer stable, but damps. Further we find that the amplitude of the localized solutions varies nearly linearly with the logarithm of the thickness of the film surrounding the localized state, larger amplitudes corresponding to a thinner film. This implies that the maximum amplitude of the localized state which can exist for given parameter values 
will depend on the noise in the system and on irregularities in the plate, since film rupture will be induced by lower noise levels and by smaller irregularities for thinner films. It will be very interesting to see whether experiments of a thin liquid film on the underside of a cooled horizontal plate will exhibit such stable stalactite-like structures.

The equation we study is

$$
h_{\tau}+\nabla \cdot\left[\left(\frac{\gamma h^{3}}{3}-\frac{B m h^{2}}{2(1+B h)^{2}}\right) \nabla h\right]+\frac{s}{3} \nabla \cdot\left[h^{3} \nabla \nabla^{2} h\right]=0
$$

Here $h(x, y, \tau)$ is the dimensionless thickness of the film; $\gamma, s$, and $B$ are positive dimensionless parameters describing the gravity, surface tension, and the Biot number (related to the rate of heat transfer at the free surface), respectively, of the system; $\tau$ is a rescaled time; and $\nabla \equiv\left(\partial_{x}, \partial_{y}\right)$. Equation (1) results from making a thin film approximation expansion of the incompressible fluid dynamics equations and assuming the surface tension is large.

This equation was derived in Refs. 11-13 and, assuming $\gamma>0, B>0$, and $s>0$, describes the spatio-temporal evolution of a thin liquid film bounded by a rigid horizontal plate of uniform temperature from above and by its free surface from below (the RayleighTaylor instability); and experiencing surface tractions arising from the surface tension dependence on the temperature (the Marangoni or the thermocapillary effect). The value of the parameter $m$ is 1 when the temperature of the plate is lower than that of the ambient air and -1 when the plate temperature is higher. A linear stability analysis shows that for $m=1$, a film of uniform thickness $h_{u}$ is stable if $h_{u}\left(1+B h_{u}\right)^{2}<(3 B) /(2 \gamma)$, demonstrating that the Marangoni effect can stabilize the Rayleigh-Taylor instability, and unstable for $h_{u}\left(1+B h_{u}\right)^{2}>(3 B) /(2 \gamma)$, provided that the domain is sufficiently large. Further, as previously noted, the Marangoni effect can result in localized nonlinear drop-like solutions in the 1 -d equation. ${ }^{13}$ For $m=-1$ the Marangoni effect is destabilizing and small unstable perturbations are found to evolve to film rupture. 
Similar to the one-dimensional case, equation (1) may be written in the form

$$
h_{\tau}=\nabla \cdot\left[h^{3} \nabla\left(\frac{\delta F}{\delta h}\right)\right] \text {, }
$$

where the Liapunov functional $F$ is

$$
F(\tau)=\int d x d y\left[P(h)+\frac{s}{6}\|\nabla h\|^{2}\right]
$$

and the potential $P$ is

$$
P(h)=-\frac{\gamma}{6} h^{2}+\frac{B m}{2} h \ln \left(\frac{h}{1+B h}\right) .
$$

In the above and below, $\|||$ denotes the vector norm, and the domain of integration is assumed to be periodic. Therefore boundary terms will vanish.

Taking $d F / d \tau$ and integrating by parts gives

$$
\frac{d F}{d \tau}=-\int d x d y h^{3}\left\|\nabla\left(-\frac{d P}{d h}+\frac{s}{3} \nabla^{2} h\right)\right\|^{2} .
$$

Since $h>0$, we have $d F / d \tau \leq 0$ and thus $F$ is strictly nonincreasing in time. We also note that $Q=\int d x d y h$ is a constant of the motion. Therefore, assuming that a stable stationary state exists, it will be given by minimizing $F$ subject to the constraint $Q$ is constant. Taking $\delta(F+\lambda Q)=0$, where $\lambda$ is a Lagrange multiplier, we find

$$
\frac{s}{3} \nabla^{2} h=\frac{d P}{d h}+\lambda
$$

for the equation satisfied by a stationary state. This equation may be solved by finding the steady-state solutions of

$$
h_{t}=\frac{s}{3} \nabla^{2} h-\frac{d P}{d h}-\lambda,
$$

where the constraint $Q$ is constant is imposed by taking

$$
\lambda(t)=-\frac{1}{\int d x d y} \int d x d y d P / d h
$$


Defining $R \equiv d P / d h-(s / 3) \nabla^{2} h$ we have $d F / d t=-\int d x d y R^{2}+\left[\int d x d y R\right]^{2} / \int d x d y \leq$ 0 . Therefore, the solutions of eq. (6) found via solving the dynamical equation (7) are not only stationary solutions of eq. (1), but are guaranteed to be stable stationary solutions since the Liapunov functional $F$ is strictly nonincreasing during the evolution of eq. (7). We stress that eq. (7) does not reproduce the transient behavior of the system, but is only a mathematical trick to find stable stationary states. If one is interested in the timedependent behavior, then it is necessary to solve eq. (1), which requires a great deal more computer time and is more difficult to solve due to the highly nonlinear nature of the equation.

By taking $m=1$, and scaling with respect to time, space, and amplitude as $\tau=$ $(8 \gamma s) /\left(9 B^{3}\right) T,(x, y)=\sqrt{s / \gamma}(X, Y)$, and $h=(3 B) /(2 \gamma) H$, eq. (1) may be written as

$$
H_{T}+\nabla \cdot\left[\left(H^{3}-\frac{H^{2}}{(1+\beta H)^{2}}\right) \nabla H\right]+\nabla \cdot\left[H^{3} \nabla \nabla^{2} H\right]=0 .
$$

where $\nabla=\left(\partial_{X}, \partial_{Y}\right)$ and $\beta=\left(3 B^{2}\right) /(2 \gamma)$. To write eqs. $(2-8)$ in the scaled variables, make the substitutions $x \rightarrow X, y \rightarrow Y, h \rightarrow H, s \rightarrow 3, \gamma \rightarrow 3, B m \rightarrow 2, B \rightarrow \beta$. We look for stable localized solutions of eq. (9) by numerically solving eqs. $(7,8)$ in the scaled variables. We used fourth-order finite differencing for the spatial derivatives, taking a 92 by 92 grid, and used the Euler method for the time integration (since we are only interested in the stationary state, the Euler method is entirely sufficient). Periodic boundary conditions were used. The accuracy of the solution was checked by evaluating $d F / d t$ from eq. (5).

Figure 1a shows a stable localized solution for $\beta=1$ which evolved from an initial Gaussian. The amplitude of this state is $A=5.7874$ and the background thickness of the film surrounding the state is $H_{b}=6.2789 \times 10^{-2}$. From eq. (8) we find $\lambda=1.9507$. Figure 1b shows a stable localized solution with amplitude different from that of fig. 1a for the same value of $\beta$ (note the change in vertical scale). For this state we have $A=13.6065$, $H_{b}=6.5057 \times 10^{-3}$, and $\lambda=4.0542$. Note that the larger amplitude state has a smaller 
background thickness. To demonstrate that stable localized solutions exist for a wide range of $\beta$ and that the form of the solutions are very similar, figs. $2 a$ and $2 b$ show stable localized states for $\beta=.01$ and $\beta=100$, respectively (again note the vertical scale). For fig. 2a we have $A=12.9896, H_{b}=6.0675 \times 10^{-2}$, and $\lambda=1.8641$. For fig. 2b we have $A=2.1863, H_{b}=3.3550 \times 10^{-3}$, and $\lambda=5.2410$. We see that the the width of the localized state is rather insensitive to the value of $\beta$.

Some insight may be gained by plotting the effective potential $P(H)+\lambda H$ as a function of $H$. Figure 3 shows such a plot for the state shown in figure 1a. We see that there is a potential well which confines the flat portion of the film surrounding the localized state. As $H$ increases, the potential increases and reaches a maximum and then decreases, approaching $-\infty$ as $H \rightarrow \infty$. In contrast to the localized states in phase dynamics ${ }^{5,6}$ where two potential wells exist, there is only one potential well here. Considering this fact, it may be surprising that stable localized states exist. However, it is the gradient term in the Liapunov functional that is responsible for the existence and stability of the state.

Figure 4 shows plots of $A$ as a function of $\log _{10}\left(H_{b}\right)$ for stable localized solutions for 5 different values of $\beta$. To produce these plots it was first necessary to determine the minimum amplitude (for each value of $\beta$ ) for which stable localized solutions exist. This was done by decreasing the amplitude of a stable state by a small amount and allowing the system to come to equilibrium. This process was repeated until at some amplitude the solution was no longer stable, but damped. Performing this process for each value of $\beta$ then determines the endpoints at the lower right of the curves. To the lower right of these endpoints no stable localized solutions exist. The next step is to gradually increase the amplitude, beginning at the right hand endpoints and allowing the system to come to equilibrium at each step. As $H_{b}$ became very small, it was necessary to decrease the time step in order to get an accurate solution (determined by evaluating $d F / d t$ ). Therefore, the upper left endpoints of the curves only reflect a finite amount of computer time and 
not the nonexistence of solutions. We expect that the curves continue to the upper left at approximately constant slope.

An important point that can be inferred from fig. 4 is that even though stable localized solutions may exist for the deterministic eq. (1) for all amplitudes greater than some minimum, in practice - at some sufficiently great amplitude - the film surrounding the localized state will become sufficiently small so that rupture will be induced by noise in the system or irregularities in the plate. This implies that there is a maximum amplitude, the value of which will be determined by the noise level or by the magnitude of the irregularities. More precisely, we would expect that the maximum amplitude would vary approximately linearly with the logarithm of the magnitude of the noise or irregularities. Even if the plate is perfectly smooth and all external vibrations are eliminated, noise-induced rupture would still result from thermal noise, so that in any physical system there will always be a maximum amplitude for which stable localized solutions can exist.

In this Letter we have shown that the two-dimensional evolution equation, describing the three-dimensional spatiotemporal evolution of the air-liquid interface of a thin film on the underside of a cooled horizontal plate, has a Liapunov functional that is strictly nonincreasing in time. We use this fact to demonstrate the existence of stable axisymmetric localized patterns for a wide range of parameter values. We have found that for a given set of physical parameters of the system there exists a continuum of possible localized structures for the amplitude larger than some critical value. The initial conditions that can lead to such states and the transient behavior prior to the formation of the states are still open questions and can only be resolved upon the efficient numerical solution of the full two-dimensional nonlinear evolution equation.

A.O. acknowledges the hospitality of the Institute for Computational Mechanics in Propulsion (ICOMP) at NASA I,ewis Research Center in Cleveland where the present 
work was done. His work is partially supported by the Israel-Mexico Energy Research Fund and Grant-in-Aid of the Government of the State of Israel. 


\section{References}

1. N.J. Zabusky and M.D. Kruskal, Phys. Rev. Lett. 15, 240 (1965).

2. O. Thual and S. Fauve, J. Phys. (Paris) 49,1829 (1988).

3. H.R. Brand and R.J. Deissler, Phys. Rev. Lett. 63, 2801 (1989).

4. R.J. Deissler and H.R. Brand, to appear Phys. Rev. A 44 (1991).

5. H.R. Brand and R.J. Deissler, Phys. Rev. Lett. 63, 508 (1989).

6. R.J. Deissler, Y.C. Lee and H.R. Brand, Phys. Rev. A 42, 2101 (1990).

7. T. Shlang and (i.I. Sivashinsky, J. Phys. (Paris) 43, 459 (1982).

8. R. J. Deissler, A. Oron and Y.C. Lee, Phys. Rev. A 43, 4558 (1991).

9. P. Rosenau and A. Oron, Phys. Fluids A 1, 1763 (1989).

10. G.I. Sivashinsky, Acta Astronaut. 4, 1177 (1977).

11. B.K. Kopbosynov and V.V. Pukhnachev, Fluid Mech.-Sov. Res. 13, 95 (1986).

12. A.D. Myshkis, V.G. Babskii, N.D. Kopachevskii, L.A. Slobozhanin and A.D. Tyuptsov, Low Gravity Fluid Mechanics, Springer-Verlag, Berlin (1987).

13. A. Oron and P. Rosenau, submitted for publication. 


\section{Figure Captions}

Fig. 1. Stable localized solutions of eq. (9) for $\beta=1$. The arrow indicates the direction of gravity. Note that similar steady solutions of different amplitudes $A$ are admitted by eq. (9) for the same value of $\beta$. (a) $A=5.7874, H_{b}=6.2789 \times 10^{-2}$, $\lambda=1.9507$; (b) $A=13.6065, H_{b}=6.5057 \times 10^{-3}, \lambda=4.0542$.

Fig. 2. Stable localized solutions of eq. (9) for various values of $\beta$ : (a) $\beta=0.01$, (b) $\beta=100$. Note the change in vertical scale.

Fig. 3. The effective potential $P(H)+\lambda H$ for the localized state shown on Fig. 1a, as a function of $H$. The value of $\lambda=1.9507$ is calculated using eq. (8). The background thickness $H_{b}=6.2789 \times 10^{-2}$ lies in the minimum of the potential well.

Fig. 4. The amplitude $A$ of the stable localized state as a function of the logarithim of the background thickness, $\log _{10}\left(H_{b}\right)$, for various values of $\beta$. Note that these curves are nearly linear. 


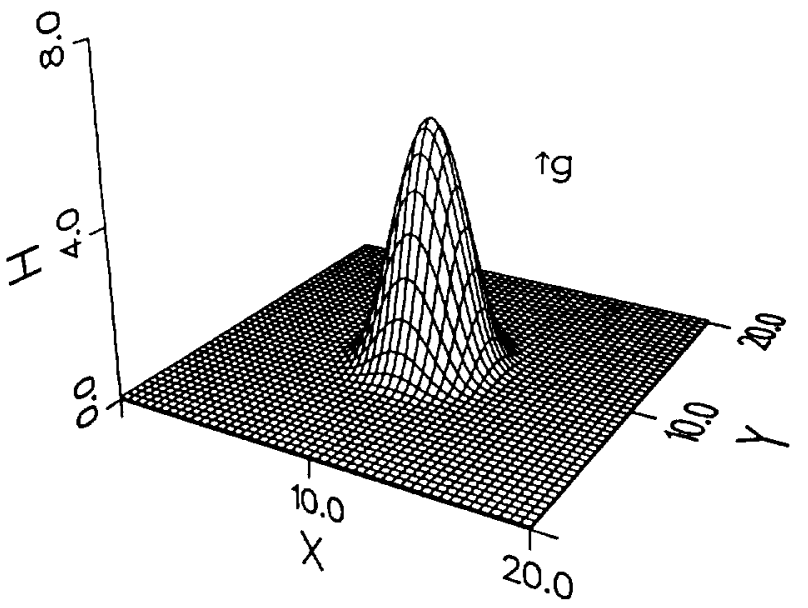

(a) $A=5.7874, H_{b}=6.2789 \times 10^{-2}, \lambda=1.9507$.

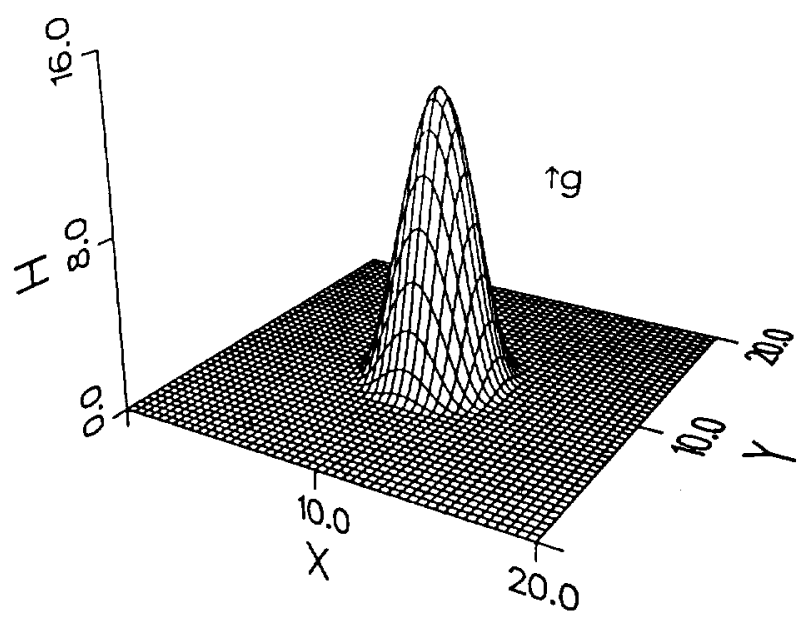

(b) $A=13.6065, H_{b}=6.5057 \times 10^{-3}, \lambda=4.0542$.

Figure 1.-Stable localized solutions of eq. (9) for $\beta=1$. The arrow indicates the direction of gravity. Note that similar steady solutions of different amplitudes $A$ are admitted by eq. (9) for the same value of $\beta$.

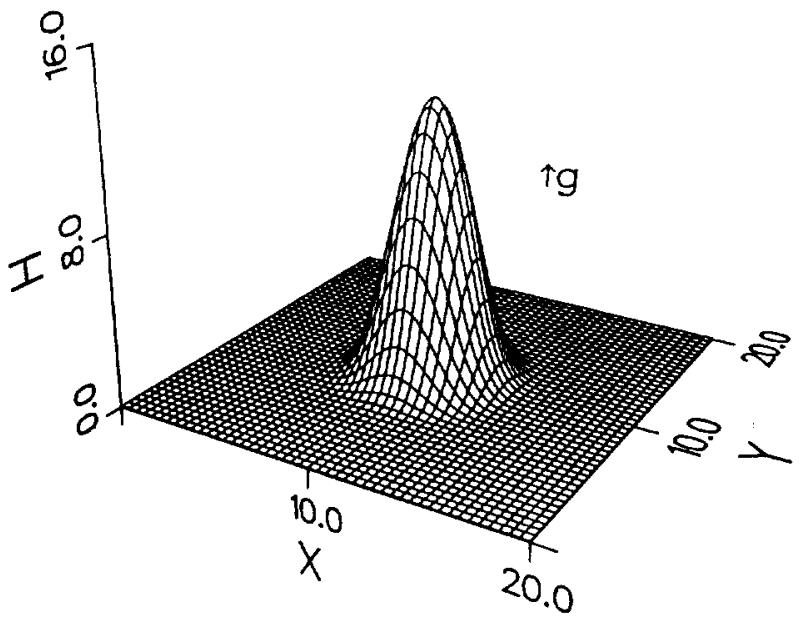

(a) $\beta=0.01$.

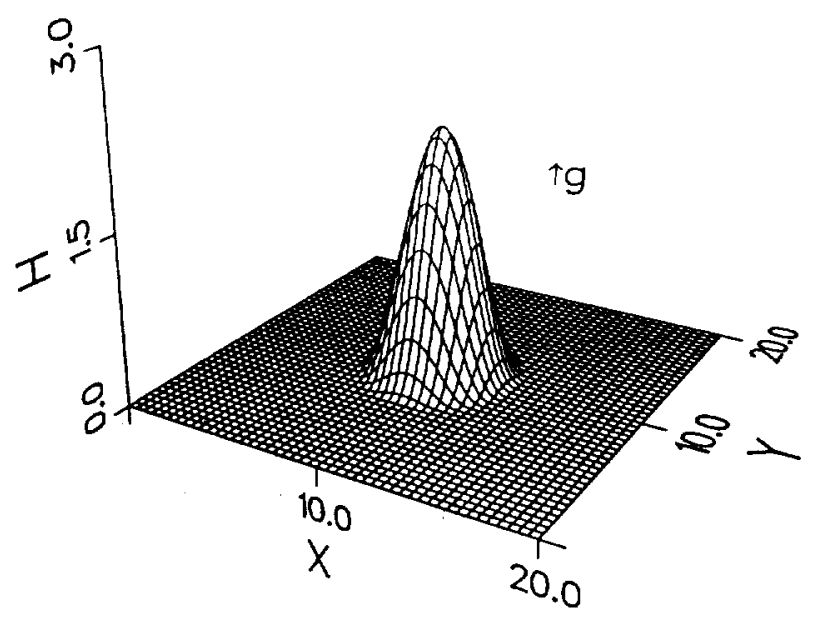

(b) $\beta=100$.

Figure 2.-Stable localized solutions of eq. (9) for various values of $\beta$. Note the change in vertical scale. 


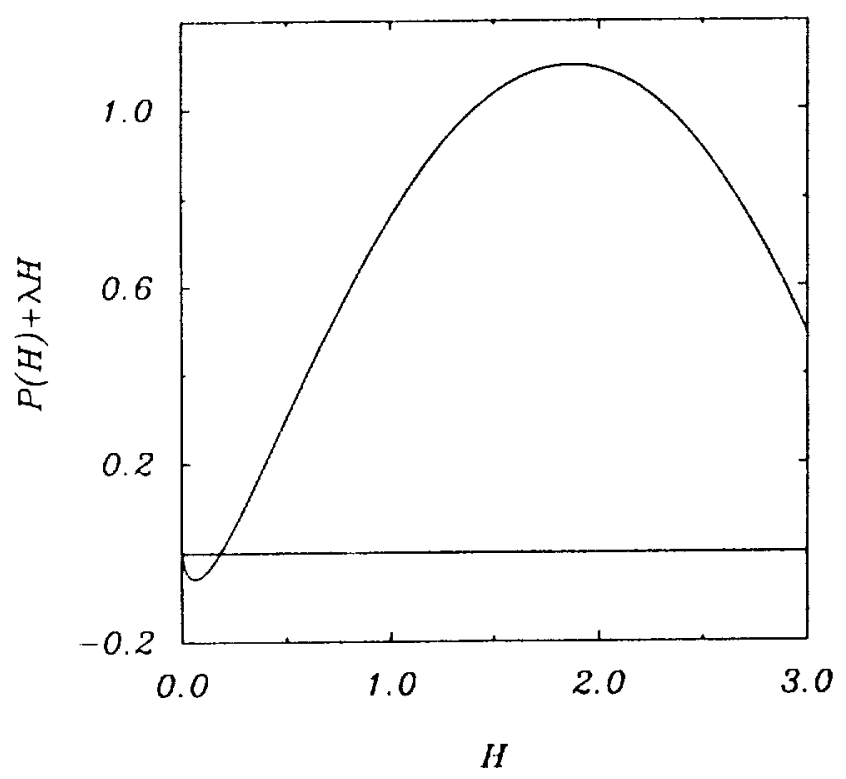

Figure 3.-The effective potential $P(H)+\lambda H$ for the localized state shown in Fig. $1 \mathrm{a}$, as a function of $H$. The value of $\lambda=1.90507$ is calculated using eq. (8). The background thickness $H_{b}=6.2789 \times 10-2$ lies in the minimum of the potential well.

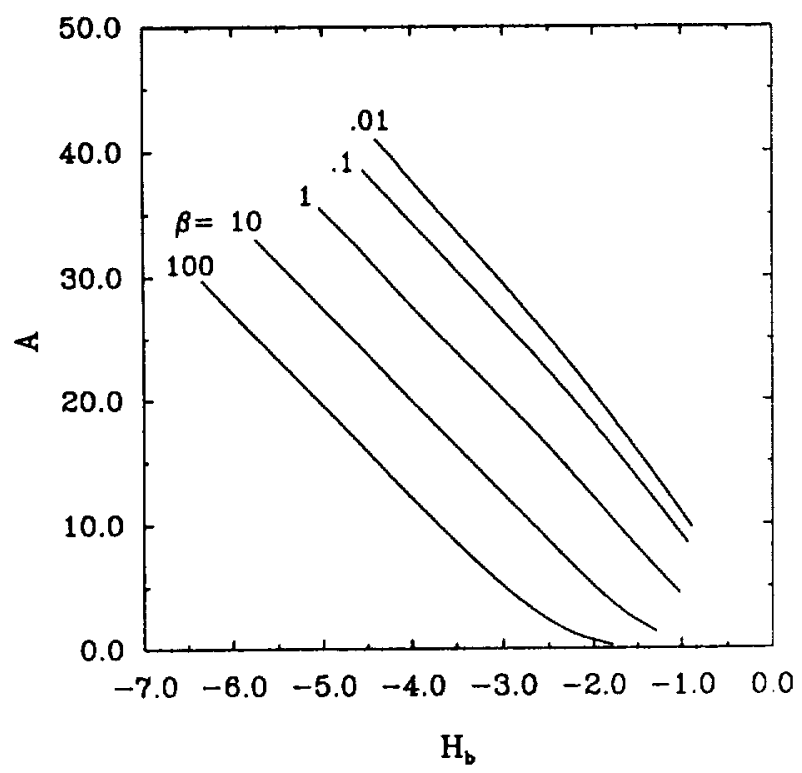

Figure 4.-The amplitude $A$ of the stable localized state as a function of the logerithm of the background thickness, $\log _{10}\left(H_{b}\right)$, for various values of $\beta$. Note that these curves are nearly linear. 
Public reporting burden lor this coilection of inlormation is estimated to average 1 hour per response, including the time for reviewing instructions, searching existing data sources, gathering and maintaining the data needed, and completing and reviewing the collection of information. Send comments regarding this burden estimate or any other aspect of this collection of information, including suggestions for reducing this burden, to Washington Headquarters Services, Directorate for intormation Operations and Reports, 1215 Jeflerson Davis Highway, Suite 1204, Arlington, VA 22202-4302, and to the Oflice of Management and Budget, Paperwork Reduction Project (0704-0188), Washinglon, DC 20503.

\begin{tabular}{|l|l|l|}
\hline 1. AGENCY USE ONLY (Leave blank) & $\begin{array}{c}\text { 2. REPORT DATE } \\
\text { November } 1991\end{array}$ & $\begin{array}{r}\text { 3. REPORT TYPE AND DATES COVERED } \\
\text { Technical Memorandum }\end{array}$ \\
\hline
\end{tabular}

4. TITLE AND SUBTITLE

5. FUNDING NUMBERS

Stable Localized Patterns in Thin Liquid Films

WU $-505-62-21$

6. AUTHOR(S)

Robert J. Deissler and Alexander Oron

7. PERFORMING ORGANIZATION NAME(S) AND ADDRESS(ES)

8. PERForming ORgANIZATION REPORT NUMBER

National Aeronautics and Space Administration

Lewis Research Center

Cleveland, Ohio 44135-3191

E-6726

9. SPONSORING/MONITORING AGENCY NAMES(S) AND ADDRESS(ES)

10. SPONSORING/MONITORING AGENCY REPORT NUMBER

National Aeronautics and Space Administration

Washington, D.C. 20546-0001

NASA TM -105352

ICOMP-91-26

11. SUPPLEMENTARY NOTES

Robert J. Deissler, Institute for Computational Mechanics in Propulsion, NASA Lewis Research Center (work funded under Space Act Agreement C-99066-G). Alexander Oron, Technion-Israel Institute of Technology, Dept. of Mechanical Engineers, Haifa 32000, Israel. Space Act Monitor, Louis A. Povinelli, (216) 433-5818.

128. DISTRIBUTION/AVAILABILITY STATEMENT

12b. DISTRIBUTION CODE

Unclassified - Unlimited

Subject Category 34

13. ABSTRACT (Maximum 200 words)

We study a two-dimensional nonlinear evolution equation which describes the three-dimensional spatiotemporal behavior of the air-liquid interface of a thin liquid film lying on the underside of a cooled horizontal plate. We show that the Marangoni effect can stabilize the destabilizing effect of gravity (the Rayleigh-Taylor instability) allowing for the existence of stable localized axisymmetric solutions for a wide range of parameter values. Various propertics of these structures are discussed.

14. SUBJECT TERMS

Liquid thin films; Marangoni effect; Rayleigh-Taylor instability; Thin film approximation; Nonlinear evolution equation

17. SECURITY CLASSIFICATION OF REPORT Unclassified

18. SECURITY CLASSIFICATION
OF THIS PAGE
Unclassified
19. SECURITY CLASSIFICATION OF ABSTRACT Unclassified
20. LIMITATION OF ABSTRACT

Star


